\title{
Comparison of ANFIS and ANN modeling for predicting the behavior of a catalytic methane reformer
}

\author{
B. Parvizi ${ }^{1 *}$, A. Khanlarkhani ${ }^{1}$, Y. Palizdar ${ }^{1}$, A.Farshad ${ }^{2}$ \\ ${ }^{I}$ Institute of Material and Energy (MERC), Karaj, Iran \\ ${ }^{2}$ Iranian Ghadir Iron \& Steel Co., Iran
}

Received January 9, 2018, Revised December 4, 2018

\begin{abstract}
Adaptive neuro-fuzzy inference system (ANFIS) and artificial neural network (ANN) were applied in modeling of methane mixed reforming in a packed bed catalytic reactor. These methods were developed by use of data collected from a methane reforming pilot plant using $\mathrm{CO}_{2}$ and steam and in process conditions near to MIDREX reforming plant in sponge Iron production. Different reaction temperatures from 700 to $1100 \mathrm{C}$ with different values of carbon dioxide, steam, hydrogen, methane and carbon monoxide, were randomly selected and used to generate around 5000 data set of input- output data. Both networks achieve quite satisfying scientific results with acceptable deviations. However, it is hard to say which one is better as they have close output values but ANN marginally outperformed ANFIS in predicting the reaction outputs by varying the inputs. The prediction performances of these models are compared. The accuracies of the two models were evaluated in terms of square correlation coefficient $\left(\mathrm{R}^{2}\right)$ and mean square error (MSE).
\end{abstract}

Keywords: Neural network, Neuro-Fuzzy, Catalytic reforming, Modeling

\section{INTRODUCTION}

Natural gas reforming reaction is one of the most important processes in oil, gas, petrochemical and steel production plants. The main process in each application is to convert hydrocarbons, mainly methane, to syngas with different $\mathrm{H}_{2}$-to- $\mathrm{CO}$ ratios. For example the ratio in an ammonia plant should be higher than 4 and in a MIDREX plant it should be 1.5-2 [1-3]. Among the variety of methods for syngas production, reforming with steam and carbon dioxide is commonly used due to its higher efficiency and cost effectiveness compared with other competing processes. This reaction takes place in a tubular reactor filled of mostly nickel alloy catalyst at around $900-1100{ }^{\circ} \mathrm{C}$ via an extremely endothermic reaction. Besides these reactions, the probability for occurrence of some undesirable reactions like production of coke will be high if the process parameters are not fixed at the optimized ones and lead to formation of hot spot and rupture of reforming tubes [1].

Main parameters for optimization of the process are: the composition of reactants which are methane, carbon dioxide, carbon monoxide, hydrogen, water and the temperature of the reaction zone. For finding the optimized region and controlling each parameter, developing a process model that describes the dynamic relations of inlet and outlet conditions is a must. Then this model can be used in a digital control system to predict the online behavior, return and keep the total system in

\footnotetext{
* To whom all correspondence should be sent: E-mail: behzadparvizi@gmail.com
}

optimized conditions [4-6].

Generally modeling methods are divided in two main groups: fundamental and empirical. The first group includes theoretical and mathematical relations and focuses on mass, energy and momentum balance and kinetics of reactions. Such methods are very useful in case of availability of mechanistic information. The second one includes data-driven models which can relate the inputoutput data in a dynamic mode via black box estimators. New digital control systems are mainly using an artificial intelligence based model which allows simulation and online prediction of system's behavior by processing online or offline inputoutput databases. Among the empirical AI methods artificial neural network (ANN) and adaptive neuro-fuzzy inference systems are the most promising ones and are applied to many real world systems [4]. These two methods have been applied in many researches and were compared. In some cases ANN was more accurate than fuzzy inference systems and in some cases vice versa [7-19].

C. Ozel used these methods for estimation of the thermal conductivity coefficient of some materials and revealed that ANFIS has less RMSE than ANN and is more accurate [7]. Areerachakul has used these two methods for estimation of biochemical oxygen demand parameter in surface waters, and as a result ANN was more accurate than the ANFIS model [8]. In another study conducted by Chauhan, these models were used in a dynamic control model for basic oxygen furnace steel making process with a higher yield for the neuro-fuzzy model [11].

The motivation behind this paper was to develop 
B. Parvizi et al.: Comparison of ANFIS and ANN modeling for predicting the behavior of a catalytic methane reformer

a method for modeling empirical relations of input (reactant composition and temperature) and output data (outlet compositions) from a pilot unit of a mixed reformer to use in online implemented control. ANN was used with BP algorithm and ANFIS by dividing data space into rule patches and the versatility, robustness and predictive accuracy of each method was compared.

\section{MODEL DEVELOPMENT Material and methods}

A pilot plant was built specifically to evaluate experimental data gathering of methane reforming with steam and carbon dioxide. The basic design of this pilot was scaled down from an industrial unit of DRI production using MIDREX technology. It essentially consists of a tubular reactor with 2" diameter and $2 \mathrm{~m}$ height, filled of three levels of catalysts, inert, semi-active and active with different percentage of nickel oxide. The reactor is fixed in a cubic electrical heater designed to reach $1400{ }^{\circ} \mathrm{C}$ with three heating zones. Five temperature sensors (TT) were implemented on inlet, outlet and three sections of the heater for accurate control of the reaction temperature. Also five mass flow controllers (model: Alicat- MCR) for gas cylinder lines and one vortex flow meter (model: YokogawaDY015) on the steam line were used for accurate control of the reactant flow rates. This plant is schematically shown in Figure 1.

Methane, steam, carbon dioxide, carbon monoxide, hydrogen and nitrogen were mixed and preheated up to around $500{ }^{\circ} \mathrm{C}$ through electrical heaters, and then the mixture flows to the reactor on three levels of catalysts. The reaction occurs at
900-1100 ${ }^{\circ} \mathrm{C}$ and produced syngas is then cooled via a water condenser and then goes online through a gas chromatograph for composition analysis. The typical operational reaction conditions and catalyst properties in the MIDREX plant used in sizing of the equipment are reported in table 1.

To know the whole process well and find out the contribution of each process parameter on outlet condition, a sufficient and most informative data set should be generated to learn the ANN and ANFIS structures, which could cover the whole data space in experimental conditions. Therefore, around 5000 sets of randomly selected input data, comprising gas compositions from 0 to $3 \mathrm{Nm}^{3} / \mathrm{h}$ and reaction temperature from 700 to $1100{ }^{\circ} \mathrm{C}$, were used to generate the input-output dataset.

\section{Neural Network Modeling}

An artificial neural network (ANN) is composed of simple calculation nodes or neurons which are connected to each other and operate in a parallel manner just like the network of neurons in the human brain. Each neuron represents an activation function with its specific output. Figure 2 shows the basic structure of the ANN [20]. In brief the method will be applied in the project in five steps:

(1) choosing proper input/output parameters;

(2) splitting the data bank into training and testing sets;

(3) creating a proper structure of neural network;

(4) training and optimizing the network architecture and

(5) performance analysis after being trained.

A schematic view of the basic structure of ANN is depicted in Fig. 2 [21].

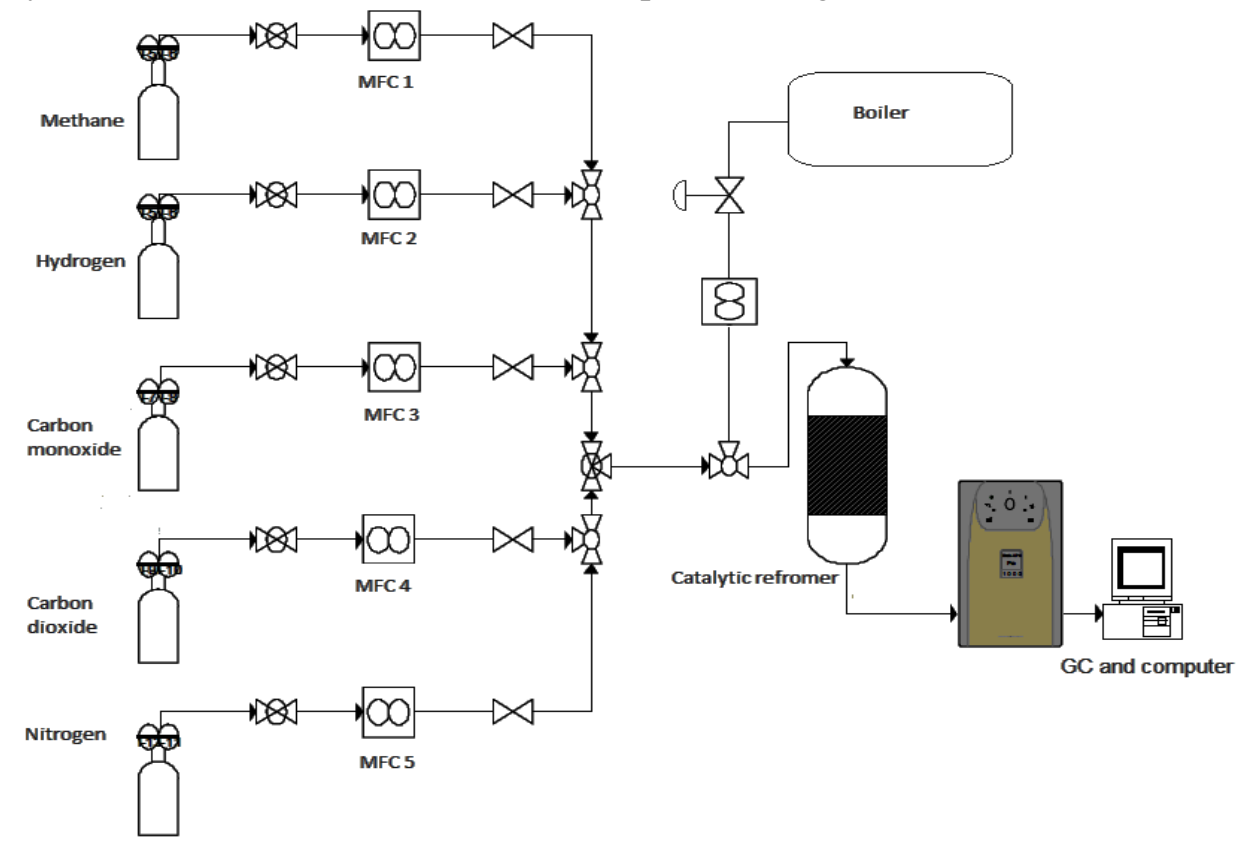

Fig. 1. Schematic diagram of the experimental system. 


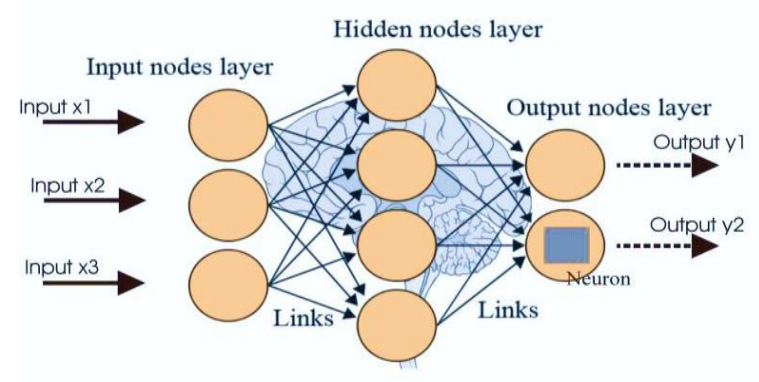

Figure 2. Basic structure of ANN

Adaptive Neuro-Fuzzy Inference System (ANFIS)

Another identification and modeling methoc is the adaptive neuro-fuzzy inference systen (ANFIS) which is a neuro-fuzzy modelin technique that applies a hybrid learning algorithm combining back propagation (BP) and leas square estimate (LSE) during the modelins process. Data-driven procedures for the construction of ANFIS networks are typically based on clustering a training set of numerica samples of the unknown function to bs approximated [22].

Table 1. Reaction conditions in the MIDREX reformer.

\begin{tabular}{|c|c|c|c|}
\hline \multicolumn{4}{|c|}{ Catalyst parameters } \\
\hline Type & $\begin{array}{l}\text { Particle size } \\
(\mathrm{mm})\end{array}$ & Porosity & Sphericity \\
\hline $\begin{array}{c}\text { Raschig } \\
\text { ring }\end{array}$ & $16 \times 6 \times 16$ & 0.52 & 0.656 \\
\hline Tortuosity & $\begin{array}{l}\text { Loose } \\
\text { density } \\
\left(\mathrm{kg} / \mathrm{m}^{3}\right)\end{array}$ & $\begin{array}{c}\text { Bed } \\
\text { density } \\
\left(\mathrm{kg} / \mathrm{m}^{3}\right)\end{array}$ & \\
\hline 2.74 & 2390 & 1362 & \\
\hline \multicolumn{4}{|c|}{ Reaction conditions } \\
\hline $\begin{array}{c}\text { Pressure } \\
\text { (bar g) }\end{array}$ & $\begin{array}{c}\text { Temperature } \\
\left({ }^{\circ} \mathrm{C}\right)\end{array}$ & $\mathrm{CO}_{2} \%$ & $\mathrm{CO} \%$ \\
\hline 2 & $900-1100$ & 15.21 & 17.4 \\
\hline $\mathrm{H}_{2} \%$ & $\mathrm{H}_{2} \mathrm{O} \%$ & $\mathrm{~N}_{2} \%$ & $\mathrm{CH}_{4} \%$ \\
\hline 31.09 & 13.94 & $1.5-2$ & 19.73 \\
\hline
\end{tabular}

Each layer in the network corresponds to a part of the fuzzy inference system (FIS) called: input fuzzification, rule inference and fire strength computation, and output defuzzification. The main advantage of this kind of representation is that the
FIS parameters are encoded as weights in the neural network and, thus, can be optimized via powerful well-known neural network learning methods.

This model is mostly suited to the modeling of nonlinear systems. Figure 3 shows a typical example of ANFIS architecture [22]. Each node in layer $\mathbf{1}$ is an adaptive node, with a node function that may be a Gaussian membership function or any other membership function. Each node in layer 2 is a fixed node labeled $\pi$, representing the firing strength of each rule. Each node in layer 3 is a fixed node labeled $\mathrm{N}$, representing the normalized firing strength of each rule. Each node in layer $\mathbf{4}$ is an adaptive node with a node function. The single node in layer 5 is a fixed node labeled $\Sigma$, indicating the overall output $(Z)$ as the summation of all incoming signals [23].

ANFIS systems are often applied in conjunction with the Takagi-Sugeno (TSK) fuzzy system, its main purpose being to utilize fuzzy modeling using measured data. In the fuzzy TSK method, for a system bearing two input parameters $X$ and $Y$ each describing a membership function, are depicted in premise and consequent parts of Fig. 3(a). Where $x$ and $\mathrm{y}$ are the input to node, $i, p, q$, and $k$ are consequence parameters resulting from the training; and $A$ and $B$ are labels of the fuzzy linguistic set [24].

\section{Modeling scenario}

Most of the time, data gathering techniques are well controlled, which results in outliers, missing values and different types of errors. Analyzing data that have not been carefully separated for these problems can produce confusing results. In order to avoid such problems, min-max normalization method was implemented to the gathered data before mathematical modeling.

A multilayer perceptron feed-forward neural network with back-propagation algorithms was used to predict the reformer behavior and syngas produced. The considered learning rule and training function are Levenberg Marquardt and trainBR, respectively. A number of around 3461 data were utilized for training session. By using the trained structure and remaining experimental data, the model was tested and the difference between the real and model data was calculated. Among structures and configurations tested, 4 hidden layers predicted the best and nearest result to the actual conditions. The log sigmoid function was employed as an activation function and 872 numbers of epochs were considered to overcome the over- and underfitting of data 


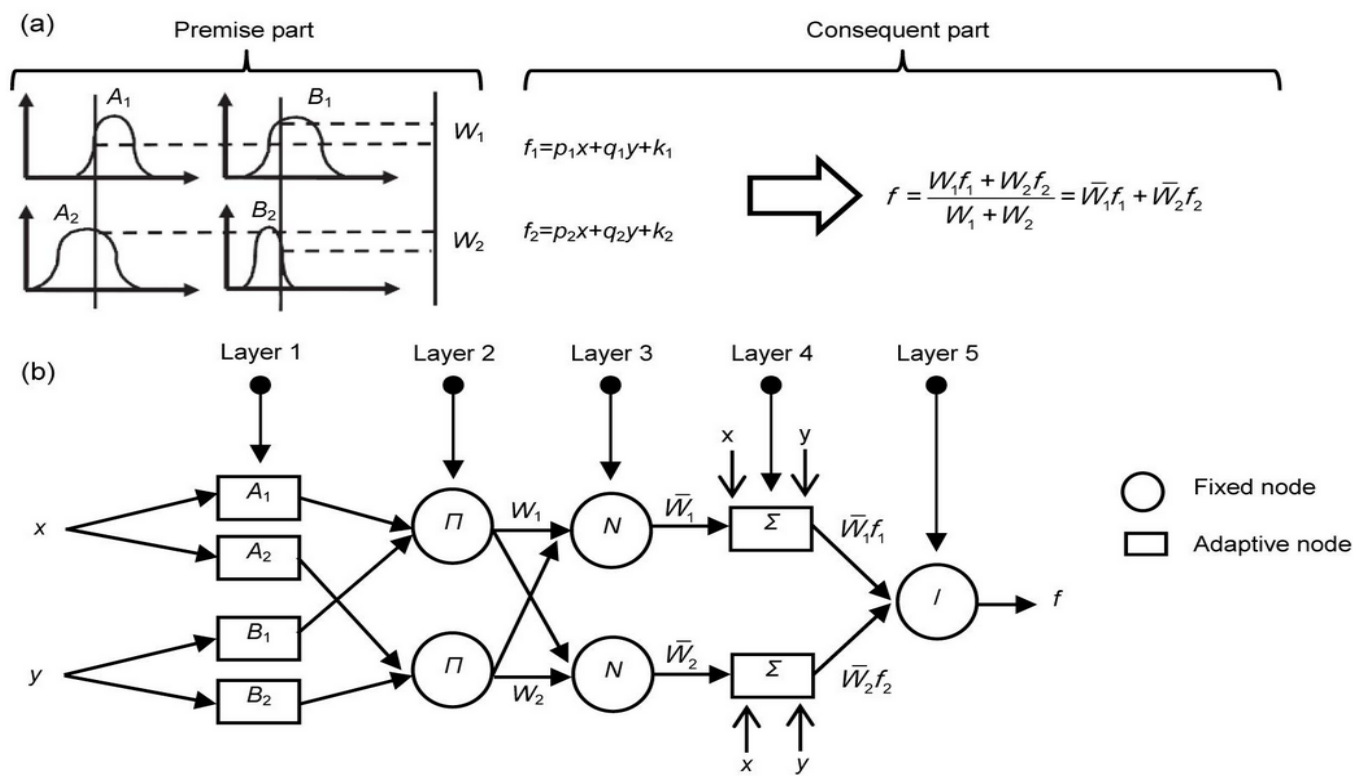

Figure 3. Basic structure of ANFIS [24].

Table 2. ANN - ANFIS model properties

\begin{tabular}{|c|c|c|c|}
\hline \multicolumn{2}{|c|}{ ANN model structure } & \multicolumn{2}{c|}{ ANFIS model structure } \\
\hline Parameter & Description & Parameter & Description \\
\hline Total layer & 6 & Total layer & 6 \\
\hline Hidden layer & 4 & Hidden layer & 4 \\
\hline $\begin{array}{c}\text { Input layer } \\
\text { neuron }\end{array}$ & 6 & Type of MF & Gaussian \\
\hline Output layer & 5 & Number of MF & 6 \\
\hline Learning rate & 0.001 & Type of output parameter & Linear \\
\hline Epoch number & 872 & Epoch numbers & $27-62$ \\
\hline Error goal & $1 \mathrm{e}^{-5}$ & Error goal & $1 \mathrm{e}^{-5}$ \\
\hline
\end{tabular}

Similar numbers of training and test data were used for the ANFIS model and tuning of the membership function of each input was performed by the hybrid method of back propagation for input membership function and the least square estimation for the parameters output membership functions. The computations of the membership function parameters are facilitated by a gradient vector which provides a measure of how well the FIS system is modeling the input/output data. The comparative properties of each model are shown in table 2.

As the most suitable method for training a neural network, the MATLAB-Simulink neural network function fitting was employed for the implementation of the ANN model. In contrast, the MATLAB command was also used to apply a fuzzy system into the forecasting model. Also the ANFIS Editor GUI applications enable the parameters of the membership functions to be automatically used. To analyse and evaluate the performance and proficiency of developed ANN and ANFIS methods in predicting the catalytic reformer two of most common error factors, $\mathrm{R}^{2}$, coefficient of determination, and MSE, mean square error, were selected and used in this work. Definition and formula of each method are denoted with the equations below:

$$
\begin{aligned}
& R^{2}=1-\frac{\sum_{i=1}^{N}\left(Y_{i}^{\text {real }}-Y_{i}^{\text {model }}\right)^{2}}{\sum_{i=1}^{N}\left(Y_{i}^{\text {real }}-Y_{a v}^{\text {real }}\right)^{2}} \\
& M S E=\frac{1}{N} \sum_{i=1}^{N}\left(Y_{i}^{\text {real }}-Y_{i}^{\text {model }}\right)^{2}
\end{aligned}
$$

where $\mathrm{N}, \mathrm{Y}(\mathrm{i})^{\text {real }}, \mathrm{Y}(\mathrm{i})^{\text {model }}$ and $\mathrm{Y}(\mathrm{av} .)^{\text {model }}$ are number of data, real data, predicted value and average of real data, respectively. It should be noted that unlike $\mathrm{R}^{2}$ which being to 1 is desirable, MSE being close to zero is desirable and shows better performance of the developed model.

\section{SIMULATION RESULTS}

In the current study, two models of ANN and ANFIS were developed by estimation of the output parameters of the catalytic reformer, which are $\mathrm{CH}_{4}$, $\mathrm{CO}_{2}, \mathrm{CO}, \mathrm{H}_{2}, \mathrm{H}_{2} \mathrm{O}$ composition, from input 
B. Parvizi et al.: Comparison of ANFIS and ANN modeling for predicting the behavior of a catalytic methane reformer parameters, which are normal flow rate of each of the above gases added by reaction temperatures. The same training and testing data sets were used to train and test both models to extract better conclusions from the comparison results. About 70 percent of the datasets were used for training of the neural network. The error histogram plot for these data is shown in figure 4 . This analysis can be an effective means for investigation and resolving the inconsistent networks performance. In this graph, blue, green and red bars represent the training, validation and testing data, respectively. As it is depicted, most of the data fall on the zero error line and there is a large central peak which indicates very small errors or outputs which are close to the target values. The performance indicators related to the training, test and validation of the ANN model including the $\mathrm{R}^{2}$ and MSE are reported in Table 3 . A comparison between the real experimental and predicted data separately is presented and discussed in the following. In parallel the ANFIS model was also implemented for input/output datasets. 6 numbers of FIS were generated. Genfis1 was used as the initial training of fuzzy sets. Training and testing process using ANFIS model were also done.

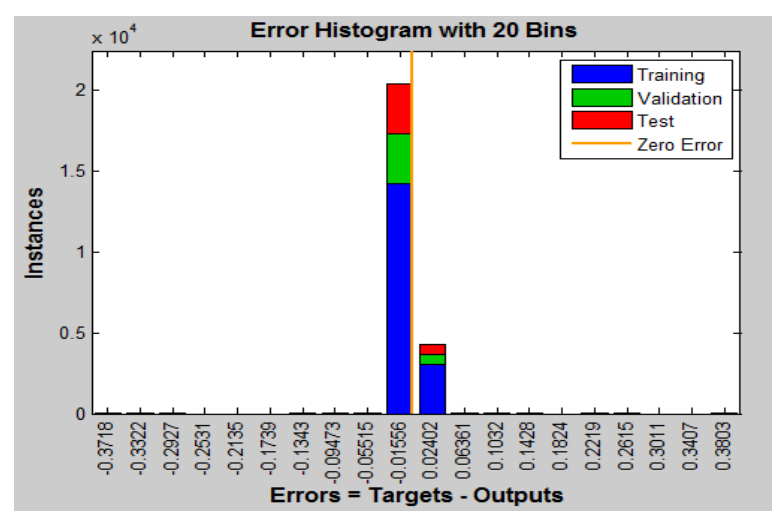

Table 3. Performance of developed ANN network

\begin{tabular}{|c|c|c|c|}
\cline { 2 - 4 } \multicolumn{1}{c|}{} & Number of data & MSE & $\mathrm{R}^{2}$ \\
\hline Training & 3461 & $7.19 \mathrm{e}^{-5}$ & $9.99 \mathrm{e}^{-1}$ \\
\hline Validation & 742 & $1.83 \mathrm{e}^{-4}$ & $9.97 \mathrm{e}^{-1}$ \\
\hline Test & 742 & $5.43 \mathrm{e}^{-5}$ & $9.99 \mathrm{e}^{-1}$ \\
\hline
\end{tabular}

Analysis of training - test in different epoch numbers was also done for each 5 different outputs in ANFIS models to find the best numbers of the epochs in each case.

Figure 5 (a-e) depicts the RMSE-Epoch numbers for all outlets. In this graph the blue line refers to training sets and green line to testing sets. In Table 4 the performance of the developed ANFIS model including MSE and $\mathrm{R}^{2}$ is reported, which can be compared with Table 3 .

Table 4. Performance of developed ANFIS model

\begin{tabular}{|c|c|c|c|}
\cline { 2 - 4 } \multicolumn{1}{c|}{} & Number of data & MSE & $\mathrm{R}^{2}$ \\
\hline Training & 3461 & $5.54 \mathrm{e}^{-5}$ & $9.99 \mathrm{e}^{-1}$ \\
\hline Validation & 742 & $5.28 \mathrm{e}^{-4}$ & $9.67 \mathrm{e}^{-1}$ \\
\hline Test & 742 & $6.68 \mathrm{e}^{-4}$ & $9.54 \mathrm{e}^{-1}$ \\
\hline
\end{tabular}

Figures 6-10 show the comparison graphs between predictions from the two networks, ANN and ANFIS against actual values obtained from the experiments. These sets of graphs are related to each outlet gas composition, respectively, methane, carbon monoxide, carbon dioxide, hydrogen and water and also each set comprised 4 sub-plots which show the comparative result of ANN with real data (a), related calculated error (b), comparative result of ANFIS with real data (c) and related calculated error (d). With regard to the huge number of input-output data, to have clearer plots, the first 200 data were considered and compared to each other.

Figure 4. Error histogram for the neural network
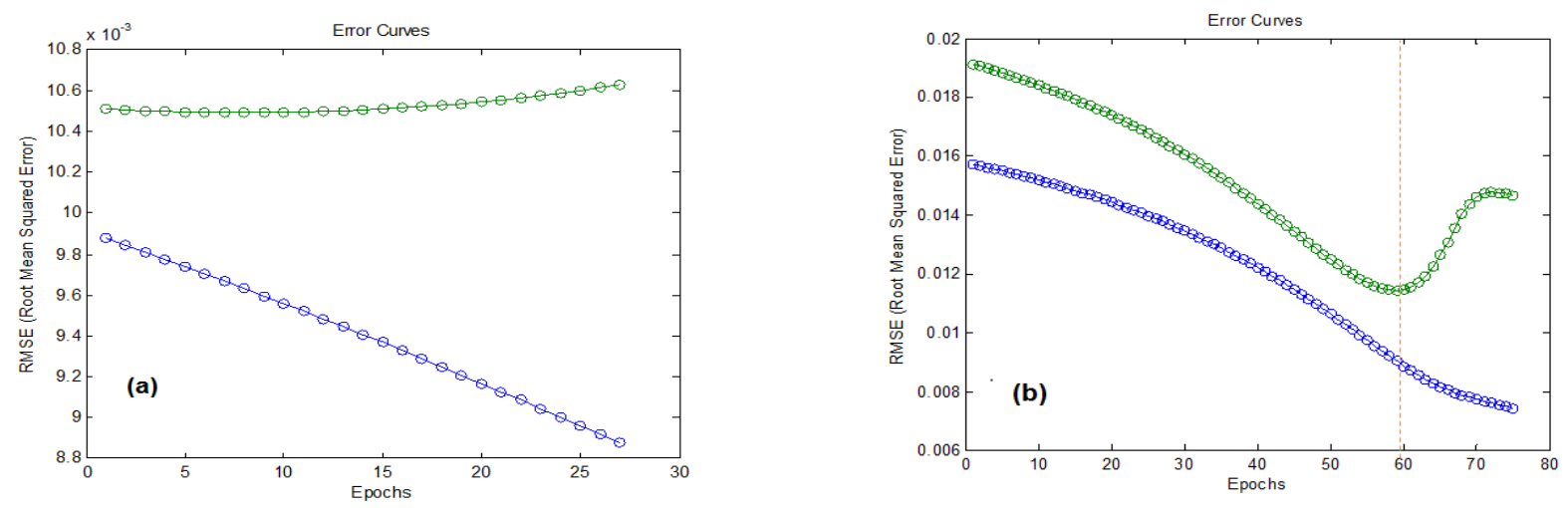
B. Parvizi et al.: Comparison of ANFIS and ANN modeling for predicting the behavior of a catalytic methane reformer
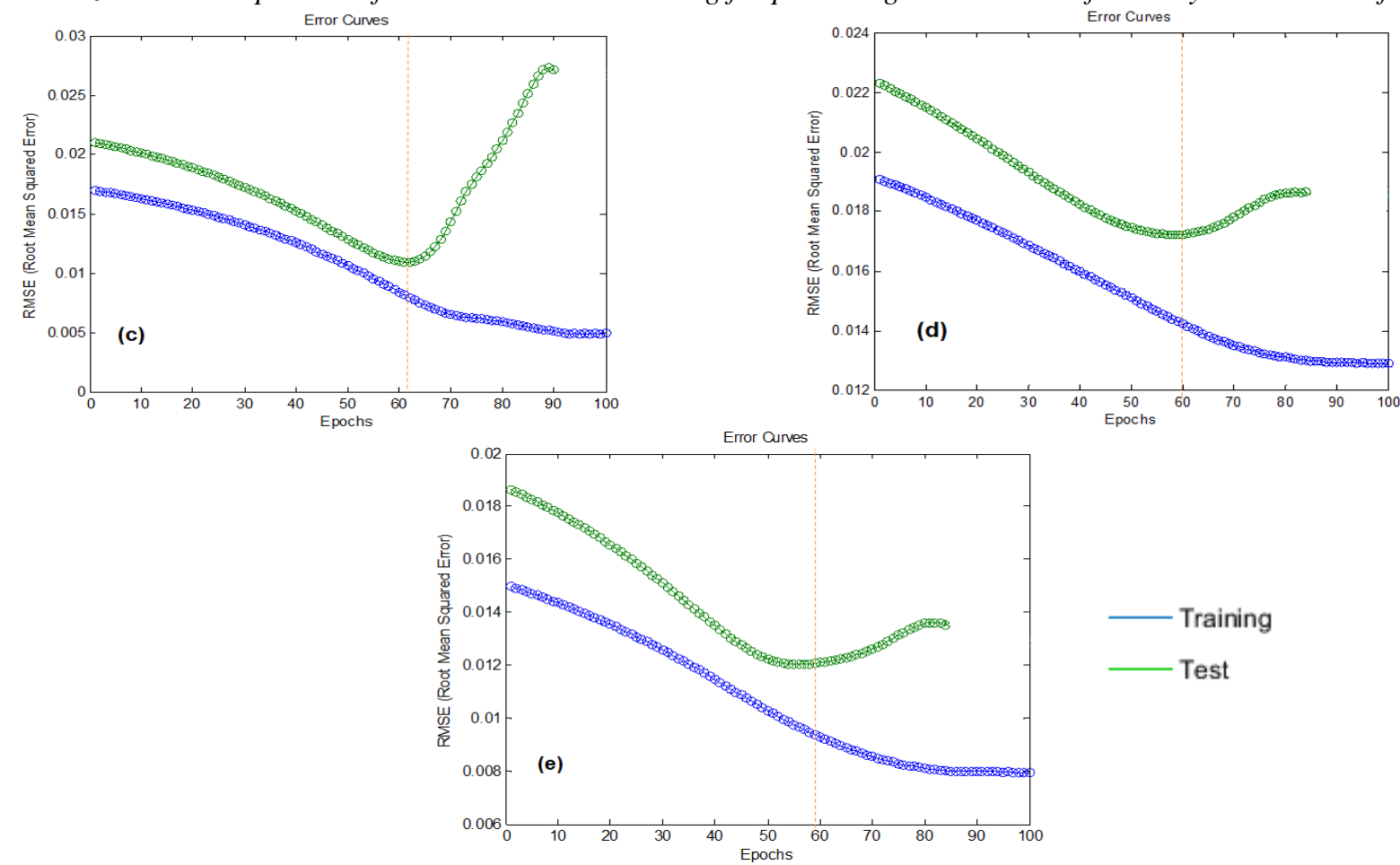

Figure 5. Test/ Train performance for ANFIS model in predicting each output and finding number of epochs. (a) $\mathrm{CH}_{4}$,

(b) $\mathrm{CO}$, (c) $\mathrm{CO}_{2}$, (d) $\mathrm{H}_{2}$, (e) $\mathrm{H}_{2} \mathrm{O}$
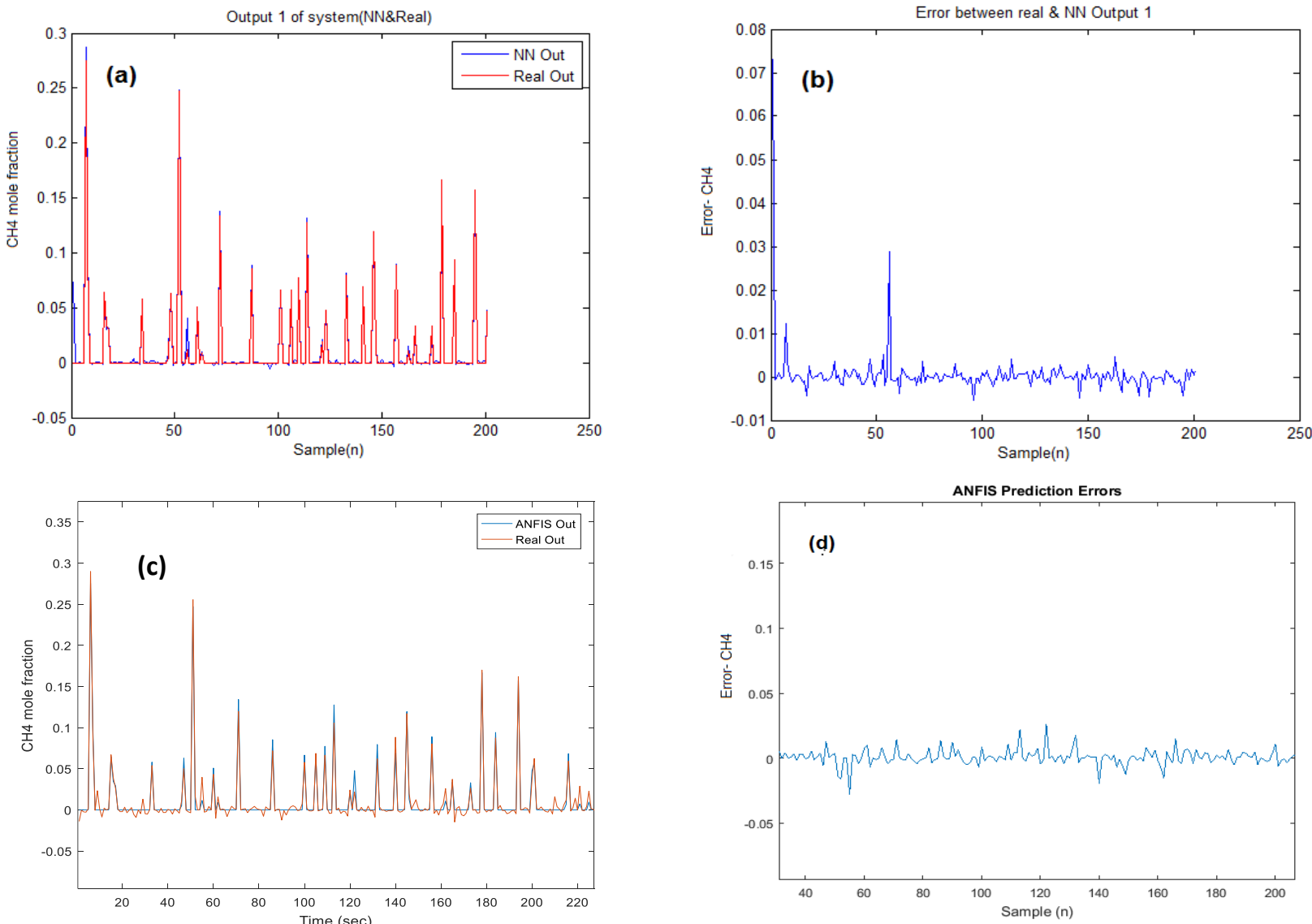

Figure 6. Comparative results for prediction performance of outlet methane composition using ANN (a,b), ANFIS $(\mathrm{c}, \mathrm{d})$ and real data

As can be seen in these graphs, there is excellent agreement between the predicted results by both ANN and ANFIS with real datasets from experimental setup. It is obvious that the ANN and ANFIS both fit and follow the real data diagram in a consistent way. Also the error plot shows the 
B. Parvizi et al.: Comparison of ANFIS and ANN modeling for predicting the behavior of a catalytic methane reformer deviation more clearly which means that all of them are in an acceptable range for both approaches.

Based on the two criteria mentioned above, $\mathrm{R}^{2}$ and MSE for the developed model, reported in tables 3 and 4 , it is obvious that, although $\mathrm{R}^{2}$ values for both models are sufficiently near to 1 , for ANN the situation is better, moreover this observation can be proven by checking the MSE. Although the

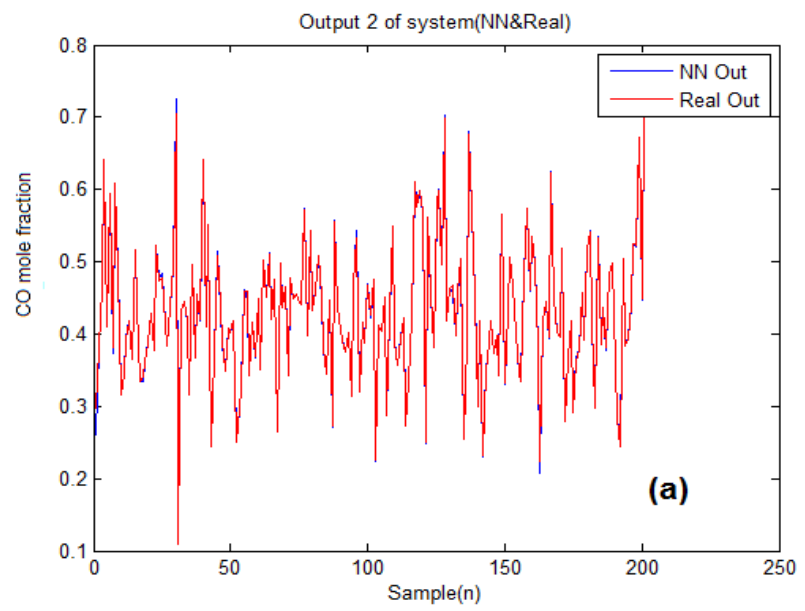
values of both are near to zero and both model outputs follow the trend of the actual ones, for ANN the values are more close to zero and so, ANN is more favorable. Despite some of its general demerits, like overfitting problems, high processing time, etc., in this special case utilizing the fuzzy logic beside the neural network did not make any added value and forward movement.
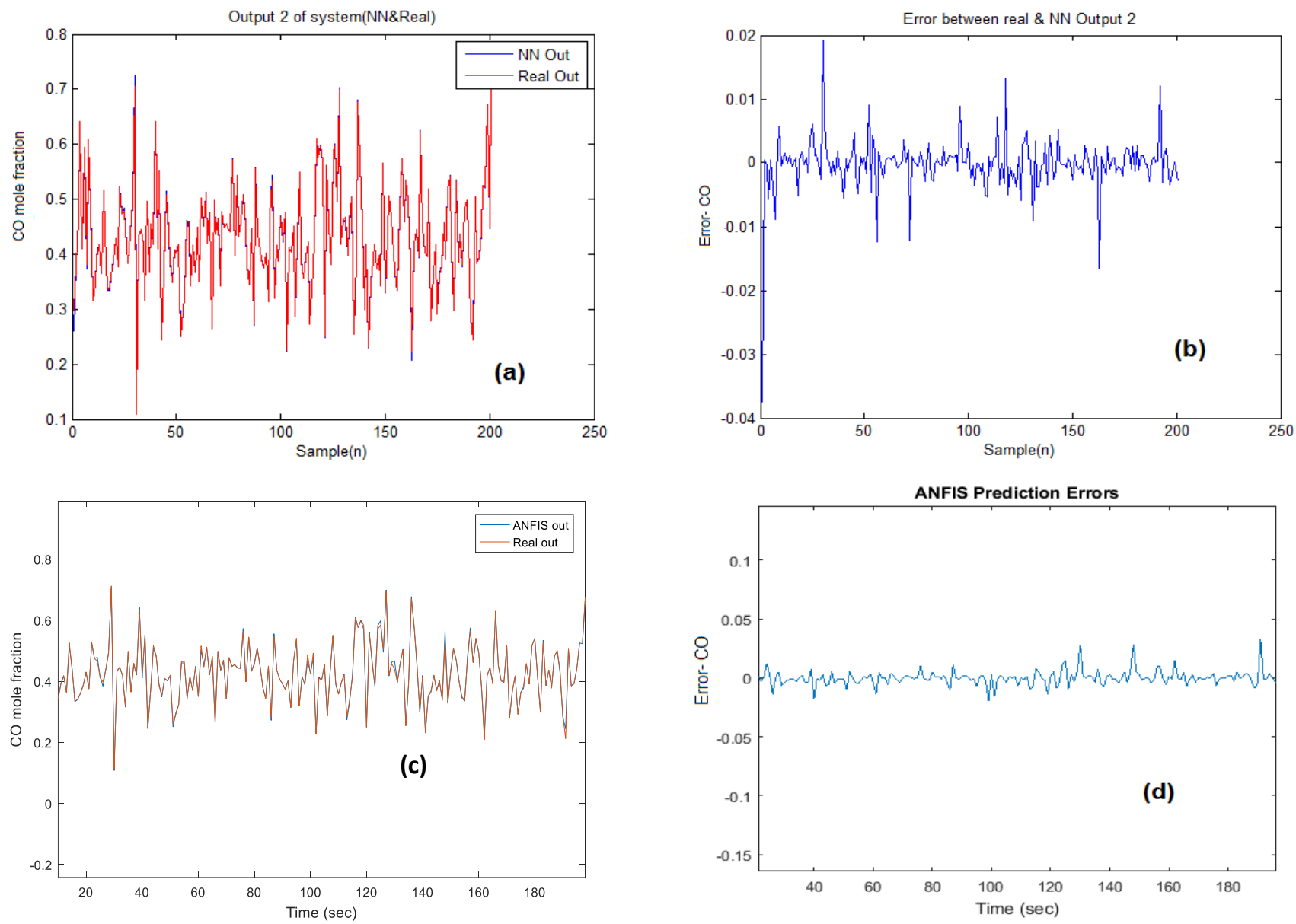

Figure 7. Comparative results for prediction performance of outlet CO composition using ANN (a,b), ANFIS $(\mathrm{c}, \mathrm{d})$ and real data. 
B. Parvizi et al.: Comparison of ANFIS and ANN modeling for predicting the behavior of a catalytic methane reformer
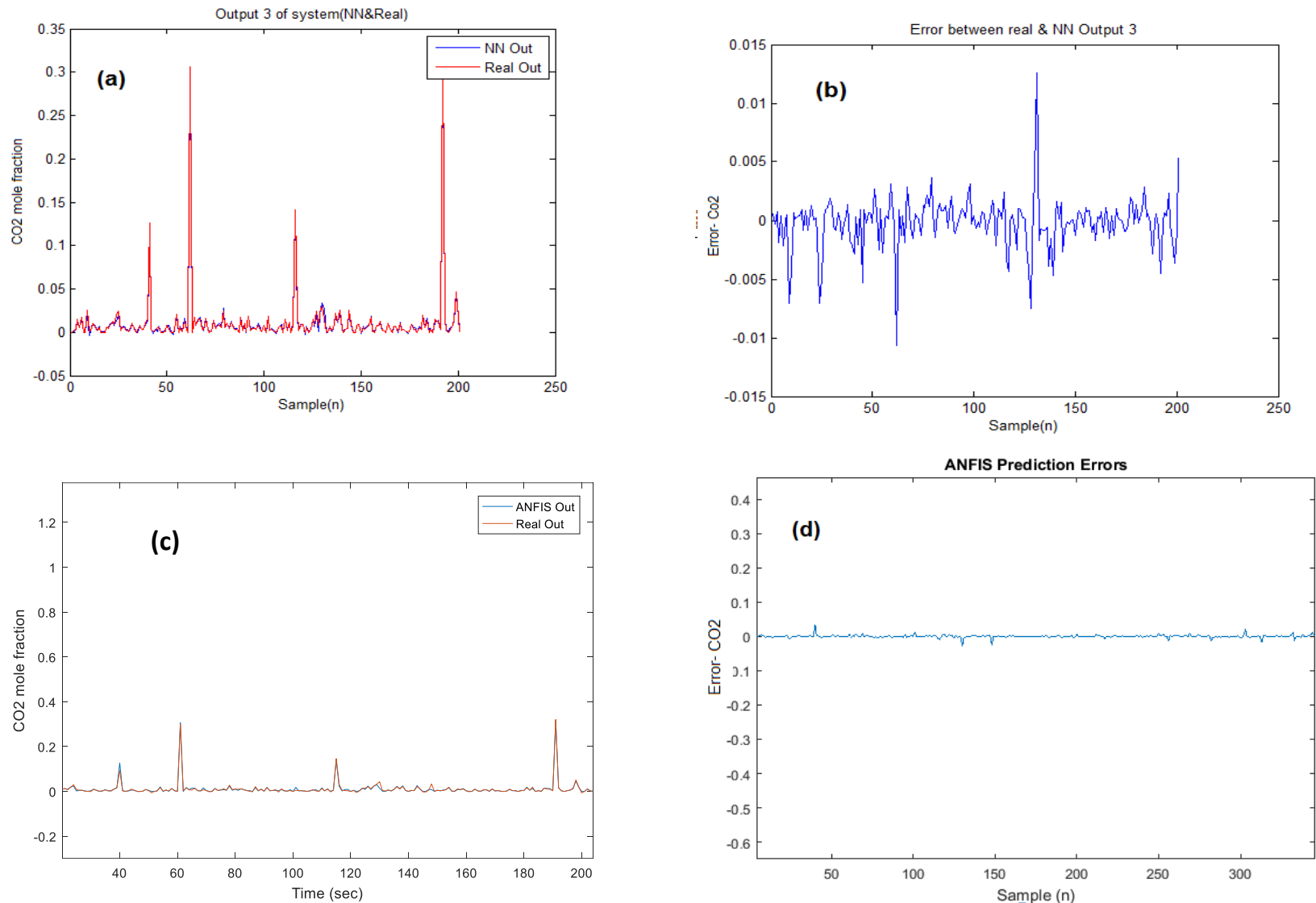

Figure 8. Comparative results for prediction performance of outlet $\mathrm{CO}_{2}$ composition using ANN (a,b), ANFIS (c,d) and real data
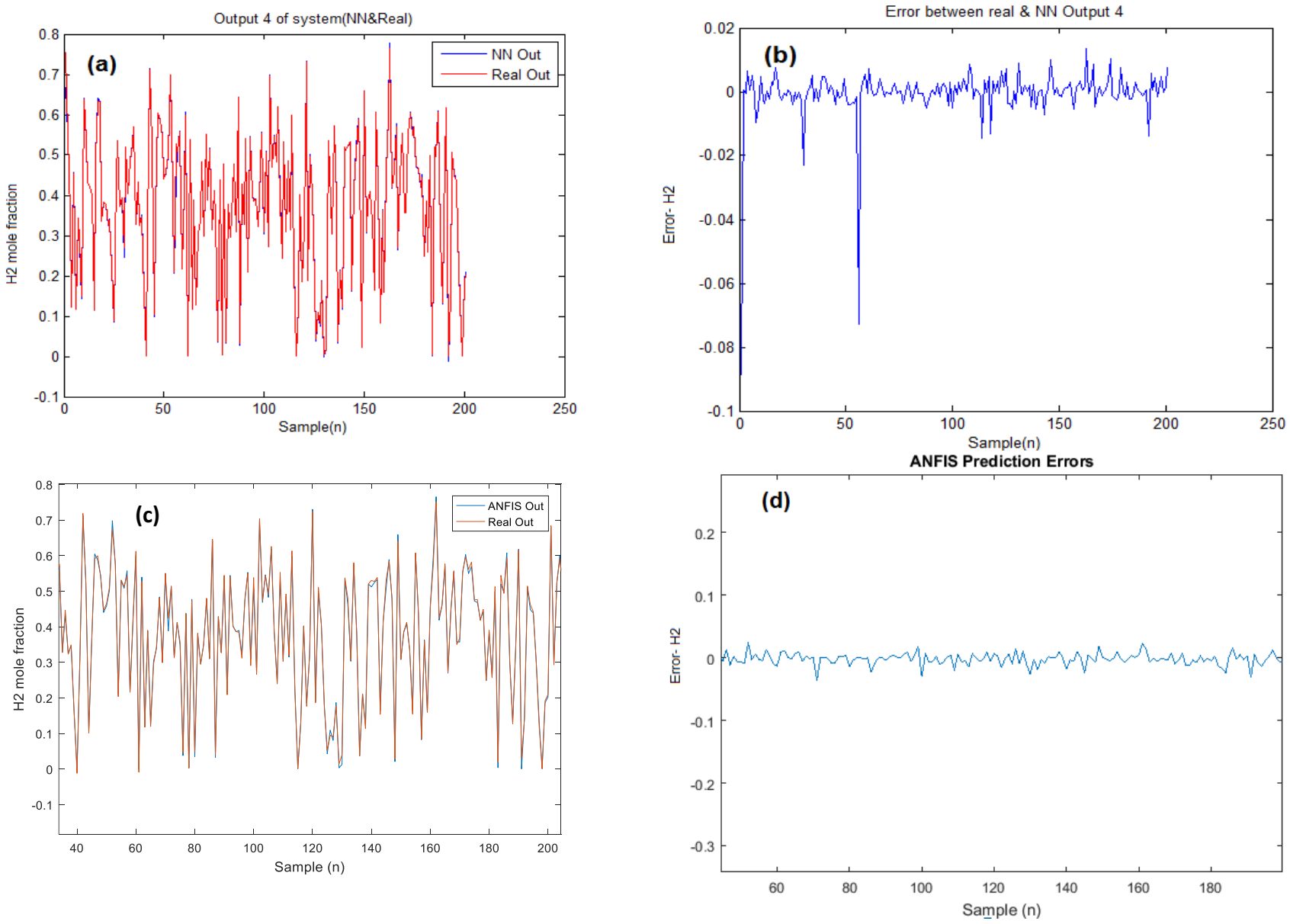

Figure 9. Comparative results for prediction performance of outlet hydrogen composition using ANN (a,b), ANFIS 

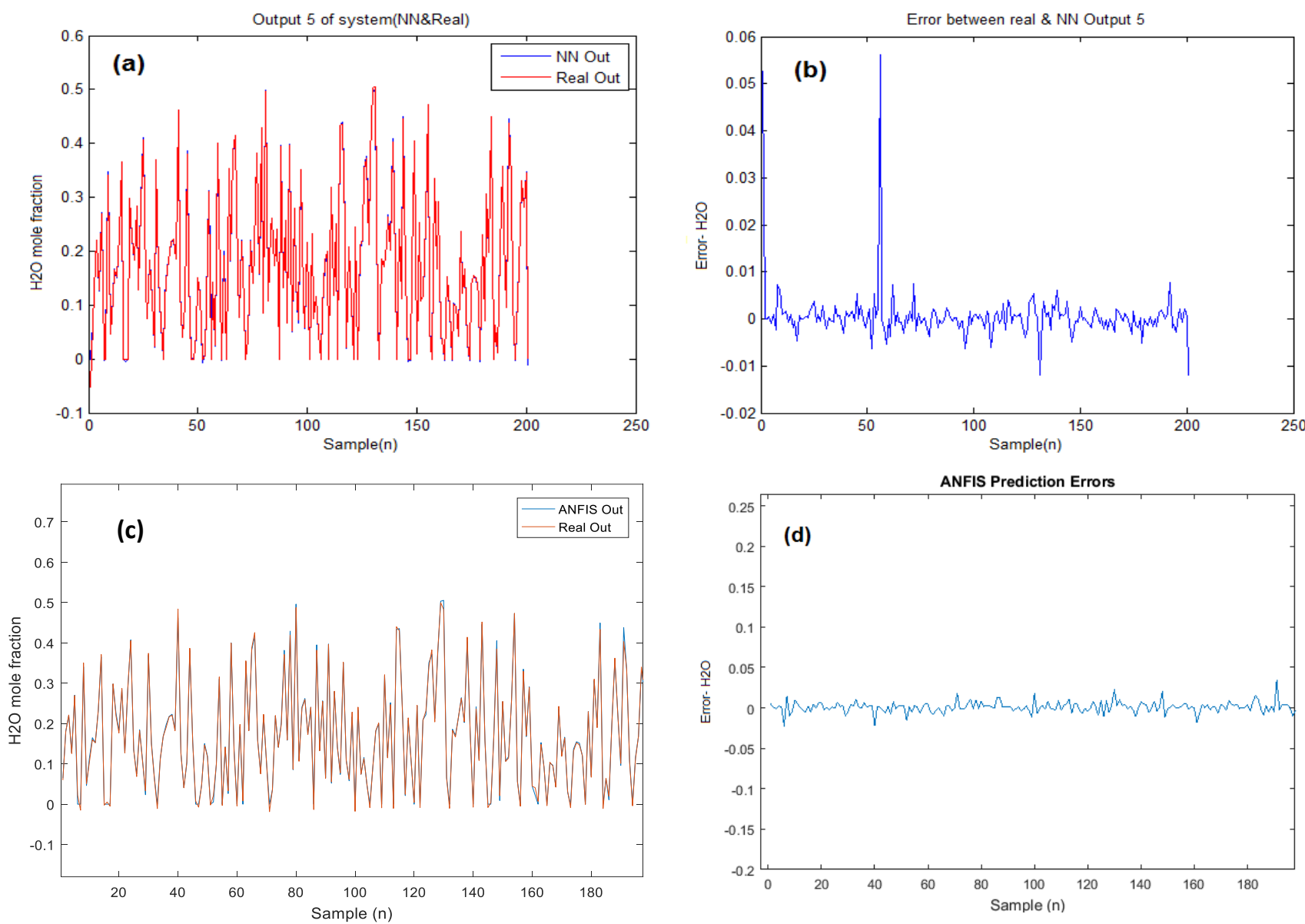

Figure 10. Comparative results for prediction performance of outlet $\mathrm{H}_{2} \mathrm{O}$ composition using ANN (a,b), ANFIS (c,d) and real data

\section{CONCLUSIONS}

Due to the importance of methane catalytic reforming for producing syngas in different industries, especially in DRI plants, which this study is focused on, for producing sponge iron, a pilot plant with the design of a MIDREX reformer was constructed. At different inlet gas flow rates and temperatures, around 5000 data were tested and their outlet data including gas compositions were acquired in an input- output databank. Two smart approaches, ANN and ANFIS were utilized for prediction of the outlet parameters from each inlet data. The predicted data were then compared with real data from the experimental ones. As to the result, both models followed the trend of real data with an acceptable accuracy and their $\mathrm{R}^{2}$ and MSE values were sufficiently near to 1 and zero, respectively. But as to these values calculated for each case, the maximum error of the ANN model was around 0.3 and this value for the ANFIS model was around 0.5, so the ANN model was more favorable and had more accurate data in comparison to addition of fuzzy system to it.

\section{REFERENCES}

1. Direct Reduction Plant, MIDREX Company Operations Manual, vol. 1, 1981, p. 8.

2. B. Cañete, C. E. Gigola, N. B. Brignole, Synthesis Gas Processes for Methanol Production via $\mathrm{CH}_{4}$ Reforming with $\mathrm{CO}_{2}, \mathrm{H}_{2} \mathrm{O}$, and $\mathrm{O}_{2}$, Ind. Eng. Chem. Res., 53, 7103 (2014).

3. A. Masaaki, MIDREX Processes, Kobelco Technology Review No. 29, 2010.

4. D. Sliškovi'c, R. Grbić, Z. Hocenski, Automatika, 52, 306 (2011).

5. M. Jajarmizadeh, S. Harun, M. Salarpour, J. Environ. Sci. Technol., 5, 249 (2012).

6. S. F. M. Jalli, S. Al-Asheh, H. E. Alfadala, Use of artificial neural network black-box modeling for the prediction of wastewater treatment plants performance, J. Environ. Manag., 83, 329 (2007).

7. C. Ozel, Scientia Iranica Transactions A: Civil Engineering, 22, 2001 (2015).

8. S. Areerachakul, Int. J. Chem. Biol. Eng., 6, 286 (2012).

9. E. K. Lafdani, A. R. Moghaddam Nia,, Int. J. Eng. Techno. Sci., 1, 32 (2013). 
B. Parvizi et al.: Comparison of ANFIS and ANN modeling for predicting the behavior of a catalytic methane reformer

10. M. K. Tiwari, S. Bajpai, Int. J. Eng. Innovative Technol. 6, 192 (2012).

11. S. Chauhan, N. K. Singh, Int. J. Eng. Innovative Technol., 2, 198 ( 2013).

12. O. Kisi, A. Murat, Comparison of ANN and ANFIS techniques in modeling dissolved oxygen", Sixteenth International Water Technology Conference, IWTC-16, Istanbul, Turkey, 2012.

13. M. T. Dastorani, A. R. Moghadamnia, J. Piri, Environ. Monitoring Assess, 166, 421 (2010).

14. P. Lotfi Anari, H. Sharifi, Res. J. Environ. Sci., 5, 236 (2011).

15. M. Aqil, I. Kita, A. Yano, J. Hydrol., 337, 22 (2007).

16. J. Kim, N. Kasabov, Neural Networks, 12, 1301 (1999).

17. T. Hussain, Z. Ali Malik, Z. Arshad, A. Nazir, J. Textile Institute, 106, 934 (2015).
18. S. Uzuner, D. Cekmecelioglu, J. BioResources, 11 8676 (2016).

19. M.T. Yilmaz, J. Texture Stud., 43, 384 (2012)

20. R. Rojas, Neural Networks, Springer-Verlag, Berlin, 1996.

21. A. Zendehboudi, X. Li, Int. J. Refrigeration, 74, 475 (2017).

22. J.-S. R. Jang, Ch.-Ts. Sun, Neuro-fuzzy modeling and control, Proceedings of IEEE, 83(3), 378 (1995).

23. A. Vasičkaninová, B. Monica, Neural Network Predictive Control and Neuro-Fuzzy Control of Heat Exchanger, Slovak University of Technology Press, 7, 2011, p. 28.

24. D. Behnia, K. Ahangari, A. Noorzad, J. Zhejiang Univ.-Sci. A, (Appl. Phys. \& Eng.), 1, 589 (2013). 